\title{
Risk Perception and Coping Strategies Among Direct Support Professionals in the Age of COVID-19
}

\author{
Johanna LoPorto, $\mathrm{PhD}$ \\ Walden University, Minneapolis, Minnesota, United States \\ (iD) https://orcid.org/0000-0002-3068-3418 \\ Kelly Spina, \\ Stony Brook University, Stony Brook, New York, Unites States \\ (iD) https://orcid.org/0000-0002-8066-6405
}

Contact: johannaloporto@gmail.com

\begin{abstract}
The spread of novel coronavirus (COVID-19) across the globe and its associated morbidity and mortality has impacted and challenged society in many ways, which resulted in adapting to a new way of life. One underrecognized and unaddressed area is the mental health of essential employees providing services to people with intellectual and developmental disabilities (IDD). Direct support professionals (DSPs) serve an important function in the daily supervision and care of clients with IDD. It is not clear, however, how these essential workers perceived their own risk of contracting COVID-19 while working during this pandemic. Our research presents results of a national survey of 478 DSPs that focused on perceptions of risk and ways of coping with COVID-19. Using an online survey, we examined DSPs' perception of risk and on the emotional and problem-solving strategies they used to cope with the global crisis. We found that DSPs engaged in higher problem-focused strategies rather than emotion-focused strategies in coping with the virus. As such, we show that it is critical for IDD provider organizations to assess DSPs' needs to provide coping supports during the age of COVID.
\end{abstract}

Keywords: direct support professionals, developmental disabilities, COVID-19

Date Submitted: January 11, 2021 | Date Published: August 27, 2021

\section{Recommended Citation}

LoPorto, J., \& Spina, K. (2021). Risk perception and coping strategies among direct support professionals in the age of COVID-19. Journal of Social, Behavioral, and Health Sciences, 15(1), 201-216.

https://doi.org/10.5590/JSBHS.2021.15.1.14

\section{Introduction}

People with IDD represent a large and diverse group, with approximately 1 in 4 adult Americans reporting some type of disability (Okoro et al., 2018). Although disability is not linked to increased risk of COVID-19 infection, associated factors or conditions may increase risks of contracting the infection for those individuals with disabilities and for those providing care. The nature of some disabilities may cause individuals difficulty in practicing preventive measures, such as people who rely on assistance with personal care and are unable to 
maintain physical distancing. Others, however, may have difficulty communicating symptoms of illness, resulting in delays in diagnosis, exposure to others, and further spread of the infection. In addition, DSPs, the essential workers who provide the care and services, may also have underlying health problems of their own (Stevens et al., 2014; Dixon-Ibarra \& Horner-Johnson, 2014), placing them at higher risk for contracting the virus.

Amid the continued spread of this infectious crisis, DSPs remain as the front-line, essential workers involved directly in the care and support for people with IDD. They are at greater risk of contracting COVID-19 and are at higher risk for psychological distress due to excessive workload, long work hours, inadequate personal protective equipment (PPE), overwhelming media news coverage, and feeling inadequately supported (Kimball et al., 2020). Although research for COVID-19 mortality and transmission rates continue, there are few studies to show how essential workers, such as DSPs, perceive the risk of infection in their work environment and on the strategies being used to cope in caring for people with IDD during the time of COVID-19.

\section{Transactional Model of Stress and Coping}

Extant literature demonstrates the various ways people cope with stress and difficult situations. Lazarus and Folkman (1984) theorized stress and coping as a transactional model that involved both cognitive and behavioral responses that people use to manage internal and external stressors. The transactional approach views stress as a process that involves continuous exchanges and adjustments known as transactions between an individual and their environment. The individual, in turn, serves as an active agent who can influence the impact of a stressor through behavioral, cognitive, and emotional strategies (Zakowski et al., 2001). Stressors, therefore, are identified by the subjective judgment of the situation that is perceived as threatening, harmful, or challenging, with responses occurring between the changeability of the stressor as experienced by the individual.

The transactional model consists of two central features. The first involves the stress being experienced, which is a cognitive process. It entails the assessment and perception by an individual in determining whether an event and/or situation threatens their well-being. The second feature follows the first, where the individual's coping response attempts to engage or change the perceived stress or threat (Lazarus \& Folkman, 1984). Coping strategies, therefore, are the actions that the individual takes in a specific situation with the intention of reducing stress that can be applied through (a) problem-focused coping strategies geared at changing the objective situation, including actions such as problem solving, gathering information, and weighing options or (b) emotion-focused coping strategies, including avoidance, minimization, distancing, and selective attention (Park et al., 2008).

For our study, the transactional model of stress and coping was found to be the most satisfactory theoretical framework to emphasize the relationships between DSPs and their work environment. We explored these essential workers' perceptions of risk while also examining the ways they coped with COVID-19.

\section{Purpose and Methodology}

The purpose of our study was to explore DSPs' perceptions of risk to COVID-19 infection while providing services to people with IDD. We also examined emotional and problem-solving coping strategies DSPs may have used within their work environments. An online Likert-scale survey, hosted on Qualtrics, was used to explore DSPs' perceptions, experiences, and coping strategies while providing services to people with IDD during the current pandemic. Wright (2005) noted that online surveys attract larger numbers of participants, while Chang and Vowles (2013) noted that online surveys are cost effective. 
In this study, we aimed to answer two research questions: (1) how do DSPs perceive the risk of being infected by COVID-19 while providing care and services to people with IDD? and (2) how do DSPs cope with the risk of being infected with COVID-19?

The participants for this study were recruited through a flyer with contact information posted online in DSPs forums and social media across all states. Data were collected for a period of 1 month, from June 23 through July 23, 2020. (Note that vaccines were not readily available in the United States and many other countries during this time period.) Participants had to be 18 years old or older and provide electronic informed consent before participating in the study. Participants were encouraged to share the flyer with other DSPs to use a snowball sampling method. This method allowed one person to refer to the next; each participant identified another potential participant to the study (Denscombe, 1998). In addition, an email account was created to allow participants to post questions on the study; however, no questions were received throughout the study.

\section{Questionnaire Development}

A 30-item online questionnaire was developed to explore DSPs' perception of the risk of infection and how they coped with that perception. A pool of 45 statements was first generated into five sections. Using this pool of statements, a panel of four DSP shift supervisors completed two reviews to select the best statements in terms of the clarity of the questions. This process decreased the number of statements to 35 in the first review. During the second review, the total was reduced to 30 statements in the final questionnaire. The front page of the online survey briefly introduced the study and a confidentiality and anonymity agreement. Once participants clicked to show their agreement to participate, the survey automatically began. Demographic questions on gender and age were included at the beginning of the questionnaire. Responses to the entire questionnaire took 10-20 minutes. To prevent missing data, we set up all questions as "required" in Qualtrics, which meant that if participants forgot to answer one question, they could not move to the next question. A total of 508 DSPs completed the survey; however, 22 DSPs' data were removed because of invalid answers. Therefore, a total of 486 DSPs fully completed the survey.

The questionnaire was divided into five sections. The first section, Risk Perception, included seven statements on DSPs' thoughts on the risk of becoming infected with COVID-19. The second section, Pandemic Perception, included eight statements on thoughts about the COVID-19 pandemic itself. The third section included five statements on the perception of experiencing fear as a result of COVID-19. The last two sections delved into DSPs' coping strategies while providing services to people with IDD during the pandemic. The fourth section included five statements on problem-focused strategies, and the final section had five statements on emotional coping strategies. Each statement required responses on a 5-point Likert scale that presented ratings from strongly agree to strongly disagree $(1=$ strongly agree, $2=$ somewhat agree, $3=$ neither agree nor disagree, 4 = somewhat disagree, $5=$ strongly disagree). This rating forced DSPs to take a position as to whether they believed they would get infected with COVID-19, if they experienced fear of the pandemic, and determined the strategies they used to cope with that perception while working as DSPs.

\section{Data Collection and Analysis}

The questionnaire was administered through the Qualtrics software and opened to participants for a period of 1 month; it was completed on two separate occasions that were 2 weeks apart. The period of 2 weeks was considered long enough for participants to have forgotten their responses but not long enough for a real change to occur in their perception of risk and COVID-19. The responses in the first administration were used in assessing construct validity and internal consistency reliability. Construct validity determines if a test measures what it is intended to measure. Reliability refers to the consistency found in repeated measurements of the same phenomenon while internal consistency refers to the extent to which all the items in the scale measure the different aspects of the same attribute. Thus, Cronbach's alpha was used to determine the reliability of the survey. Cronbach's alpha ranges from o to 1.o. The closer the Cronbach's alpha coefficient is 
to 1.o, the greater the internal consistency of the survey items will be. A cut-off of 0.70 has been used to assess the internal reliability of each scale in this study.

\section{Results}

From June 23 through July 23, 2020, 508 DSPs connected to the Internet tool, and 486 identified themselves as eligible for participating in the study (90.53\% female, 8.85\% males, and $0.62 \%$ identified as a different gender). The results indicate women as the greatest gender for providing support and care to people with IDD (Table 1).

Table 1. Participant Gender $(n=486)$

\begin{tabular}{|l|l|l|}
\hline Preferred Gender & Responses & Percentage (\%) \\
\hline Female & 440 & 90.53 \\
\hline Male & 43 & 8.85 \\
\hline Androgynous & 0 & 0.00 \\
\hline Bigender & 0 & 0.00 \\
\hline Gender fluid & 1 & 0.21 \\
\hline Nonbinary & 2 & 0.41 \\
\hline Pangender & 0 & 0.00 \\
\hline Trans & 0 & 0.00 \\
\hline Trans person & 0 & 0.00 \\
\hline Transsexual & 0 & 0.00 \\
\hline Transgender & 0 & 0.00 \\
\hline Other & 0 & 0.00 \\
\hline Total & $\mathbf{4 8 6}$ & $\mathbf{1 0 0}$ \\
\hline
\end{tabular}

The data also showed 478 (99.70\%) participants completed the age range of the survey; $28 \%$ were between 30 and 39 years old. At least eight DSPs did not provide their age (Table 2).

Table 2. Participant Age

\begin{tabular}{|l|l|l|}
\hline Participant Age Range (years) & Responses & Percentage (\%) \\
\hline $0-19$ & 5 & 1.00 \\
\hline $20-29$ & 116 & 24 \\
\hline $30-39$ & 134 & 28 \\
\hline $40-49$ & 101 & 21 \\
\hline $50-59$ & 92 & 19 \\
\hline $60-69$ & 28 & 6 \\
\hline $70-79$ & 1 & 0 \\
\hline Unreadable response & 1 & 0 \\
\hline Total & $\mathbf{4 7 8}$ & $\mathbf{9 9 . 7 0}$ \\
\hline
\end{tabular}

Participants from 40 states completed the questionnaire, with most responses coming from New York with 107 (22.92\%), followed by Ohio with 37 (7.92\%), Pennsylvania with 35 (7.28\%), Indiana with 25 (5.25\%), and 
New Jersey at 17 (3.64\%). There were 467 responses identifying states, with four non-replies and 19 who did not wish to identify their state of residence.

\section{Table 3. Participant Demographics}

\begin{tabular}{|c|c|c|}
\hline States & Responses & Percentage (\%) \\
\hline Arkansas & 2 & 0.42 \\
\hline Arizona & 1 & 0.20 \\
\hline California & 10 & 2.14 \\
\hline Canada & 6 & 1.28 \\
\hline Colorado & 6 & 1.28 \\
\hline Connecticut & 12 & 2.56 \\
\hline Delaware & 1 & 0.20 \\
\hline Florida & 11 & 2.35 \\
\hline Iowa & 10 & 2.14 \\
\hline Illinois & 15 & 3.20 \\
\hline Indiana & 25 & $5 \cdot 35$ \\
\hline Kansas & 5 & 1.00 \\
\hline Kentucky & 16 & 3.40 \\
\hline Massachusetts & 8 & 1.71 \\
\hline Maine & 12 & 2.56 \\
\hline Maryland & 7 & 1.49 \\
\hline Michigan & 10 & 2.12 \\
\hline Minnesota & 11 & 2.35 \\
\hline Missouri & 11 & 2.35 \\
\hline Montana & 2 & 0.42 \\
\hline North Carolina & 6 & 1.28 \\
\hline North Dakota & 5 & 1.07 \\
\hline Nebraska & 7 & 1.49 \\
\hline New Hampshire & 5 & 1.07 \\
\hline New Jersey & 17 & 3.64 \\
\hline New York & 107 & 22.91 \\
\hline Ohio & 37 & 7.92 \\
\hline Oklahoma & 5 & 1.07 \\
\hline Oregon & 10 & 2.14 \\
\hline Pennsylvania & 35 & 7.28 \\
\hline Rhode Island & 3 & 0.64 \\
\hline South Dakota & 6 & 1.28 \\
\hline Tennessee & 14 & 2.99 \\
\hline Texas & 2 & 0.42 \\
\hline Utah & 3 & 0.64 \\
\hline Virginia & 9 & 1.92 \\
\hline Vermont & 1 & 0.20 \\
\hline
\end{tabular}




\begin{tabular}{|l|l|l|}
\hline States & Responses & Percentage (\%) \\
\hline Washington & 2 & 0.42 \\
\hline West Virginia & 3 & 0.64 \\
\hline Wisconsin & 2 & 0.42 \\
\hline Wyoming & 3 & 0.64 \\
\hline Non-replies & 4 & 0.85 \\
\hline Total & 467 & 99.45 \\
\hline
\end{tabular}

After the demographic collection of the participants, the survey questions were then broken into four main categories: Risk Perception, Pandemic Perception, Fear of Infection, and Coping Strategies (emotion-based or problem-solving-based). The last section of the survey included an area for participants to leave comments. Participants answered closed-ended questions using a 5-point Likert scale to answer the two research questions: (a) how do DSPs perceive the risk of being infected by COVID-19 while providing care and services to people with IDD? and (b) how do DSPs cope with the risk of being infected with COVID-19 and with the pandemic?

\section{Risk Perception}

Seven questions were asked as to Risk Perception. The data showed that 365 (83.53\%) DSPs believed that they were at risk of becoming infected with COVID-19, while 329 (75.46\%) DSPs believed they could become infected simply because of their working environment. The data also showed that 339 (77.94\%) DSPs agreed that they were putting themselves at risk for becoming infected by simply coming into work, while 25 (7.44\%) indicated that they themselves were impacted by COVID-19 by contracting the virus. In addition, 390 (89.86\%) DSPs believed the people they supported were more at risk of becoming infected and 61 (18.27\%) already supported a person who had contracted the infection. Last, 191 (56.68\%) indicated that they already knew someone outside of work diagnosed with COVID-19. Cronbach alpha in this section was valued at .72, indicating good internal reliability.

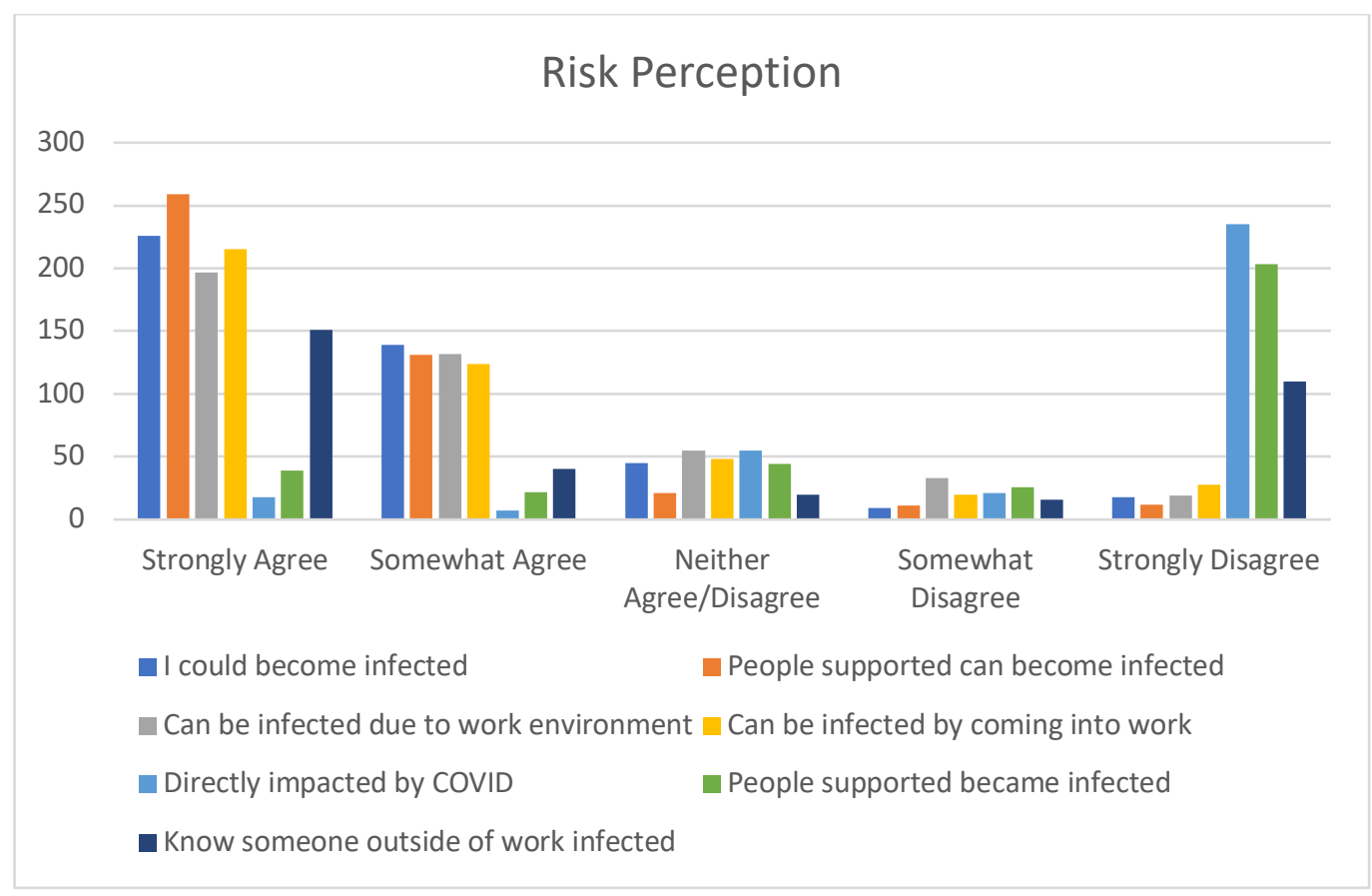

Figure 1: Risk Perception of DSPs, 5-point Likert Scale ( $1=$ Strongly Agree, $5=$ Strongly Disagree), N = 486 


\section{Pandemic Perception}

As to pandemic perceptions, DSPs were asked how they viewed the COVID-19 crisis overall. Data showed that 372 (90.29\%) DSPs strongly agreed or somewhat agreed that the COVID-19 infection was a global crisis. Data also showed that 353 (85.06\%) believed the virus would continue to cause many deaths. At least 83 (19.95\%) DSPs agreed or somewhat agreed that COVID-19 was basically "fake news," while 202 (72.25\%) DSPs believed that COVID-19 was not going to dissipate and would continue impacting their health and safety. In addition, 302 (73.14\%) DSPs agreed or somewhat agreed that COVID-19 was not going to dissipate and would impact people with IDD, and 195 (46.99\%) believed that people were at higher risk of contracting the virus because they have IDD. Finally, 256 (61.39\%) agreed or somewhat agreed that COVID-19 was impacting them personally. Originally, a third statement was included in this section asking participants how they could reduce the risk of infection for themselves and others, but the analysis suggested that this item should be removed as it did not measure the same construct as the others. Thus, removing the third statement increased Cronbach's reliability from .64 to .73 .

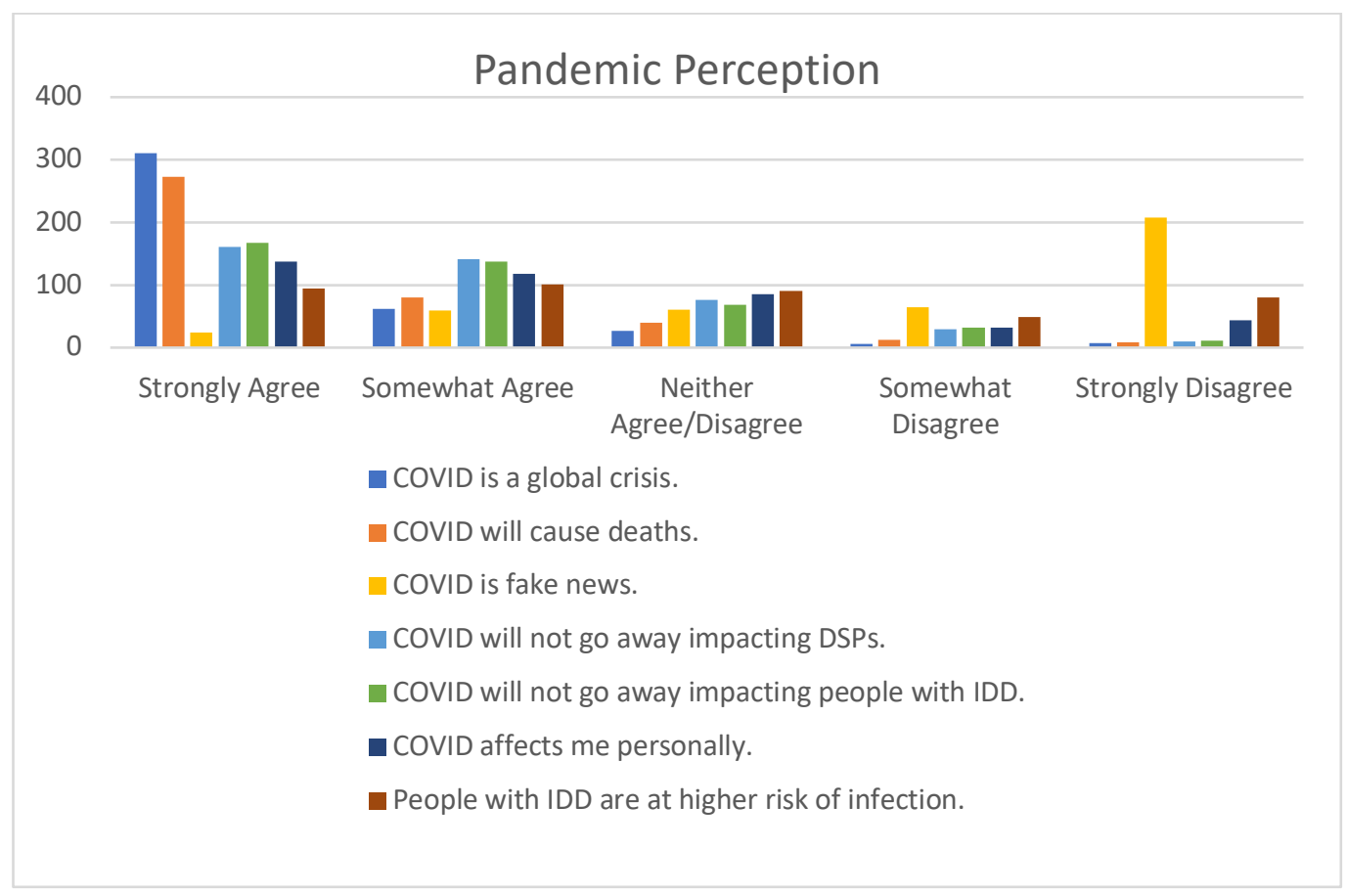

Figure 2: Pandemic Perception of DSPs, 5-point Likert Scale ( $1=$ Strongly Agree, $5=$ Strongly Disagree), $\mathrm{N}=$ 486

\section{Fear of Infection}

In examining DSPs' fear of contracting COVID-19, five questions were in the survey related to the perception of fear. The survey showed that 249 (62.88\%) worried all the time about contracting the infection. Moreover, 299 (75.50\%) DSPs feared that COVID-19 would dissipate over time only to reemerge again at some point. Other DSPs experienced less fear of contracting COVID-19 as they felt safe while at work and while providing services to people with IDD. Data showed that 222 (55.78\%) felt safe at work; 270 (67.67\%) related they had the proper PPE to do their jobs safely; and 272 (68\%) indicated they were given the tools, training, and information to reduce risk of infection at work. To meet Cronbach's alpha, items 3, 4, and 5 were reverse- 
coded to measure fear of infection at the same direction. When they were reversed, Cronbach's reliability was at .73.

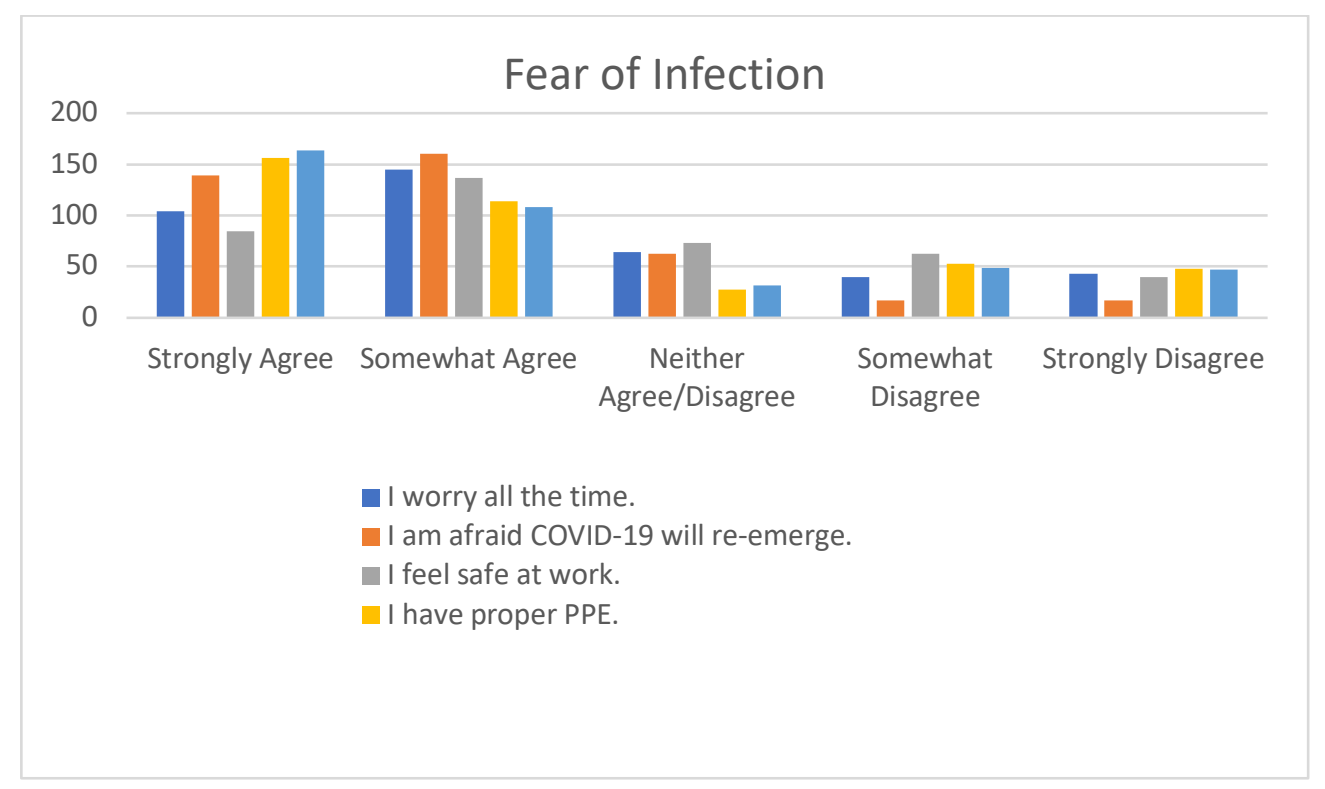

Figure 3: Fear of infection, 5-point Likert Scale ( $1=$ Strongly Agree, $5=$ Strongly Disagree), N = 486

\section{Problem-Focused Coping Strategies}

In this survey, DSPs were also asked questions to determine if problem-focused coping strategies were undertaken. Problem-focused coping strategies are geared at changing the objective situation, such as gathering information, weighing options, and acting upon choice (Holahan \& Moos, 1987). Five questions were asked in this segment with results showing that DSPs felt confident as to the COVID-19 information provided so they could act accordingly. This study showed that 309 (81.31\%) DSPs agreed or somewhat agreed to listening to experts for COVID-19 advice while 339 (88.51\%) DSPs reported they knew what to do to reduce the risk of infection for themselves and others. In addition, 251 (62.60\%) DSPs felt comfortable talking to their supervisors about COVID-19 and 363 (95.53\%) DSPs understood how to reduce the risk of infection for themselves. Finally, 357 (94.45\%) DSPs reported understanding procedures to reduce risk of infection for the people they supported. 


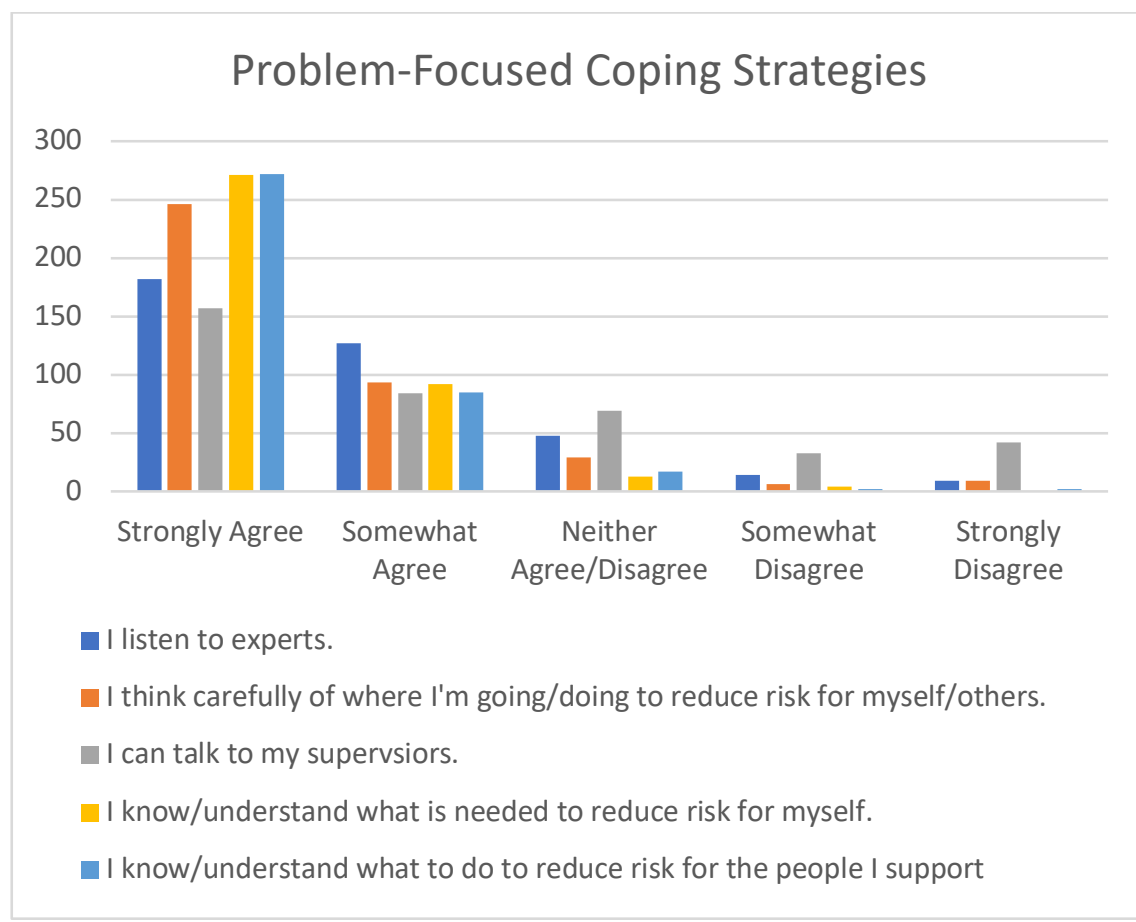

Figure 4: Problem-focused strategies of DSPs coping with COVID-19, 5-point Likert Scale (1 = Strongly Agree, 5 = Strongly Disagree), $\mathrm{N}=486$

\section{Emotion-Focused Coping Strategies}

In this segment of the study, DSPs were asked to rate emotion-focused coping strategies that were undertaken while providing services to people with IDD. The results for these questions on the survey reflect that DSPs also engaged in emotionally focused strategies in coping with COVID-19. The results showed that 132 (36.06\%) agreed or somewhat agreed that COVID-19 will dissipate over time and there was nothing they could do. The survey also prompted DSPs to respond to their belief in fate, with results showing 96 (26.16\%) DSPs agreed to submit to fate and that it was only a matter of time before they would become infected with COVID-19 while 160 (43.59\%) DSPs disagreed, believing they had some control over contracting the virus. Regarding DSPs' perception of the people they supported becoming infected, 115 (31.17\%) neither agreed nor disagreed that, over time, the people they supported could become infected. Moreover, 124 (33.60\%) DSPs believed that, in time, the people they supported would become infected; 130 (35.23\%) DSPs disagreed.

In addition, 280 (75.68\%) DSPs disagreed that they had no control over work processes, which have remained the same, indicating that organizations made changes in work processes to control and reduce the risk of infection of COVID-19 while 144 (38.92\%) agreed to making themselves feel better by simply not thinking about the COVID-19 crisis. In this section, Cronbach's alpha measured at .57 showing this survey section having poor internal reliability. Removing one item did not improve reliability indicating that this survey section collectively was not a good measure of emotion-focused coping strategies. 


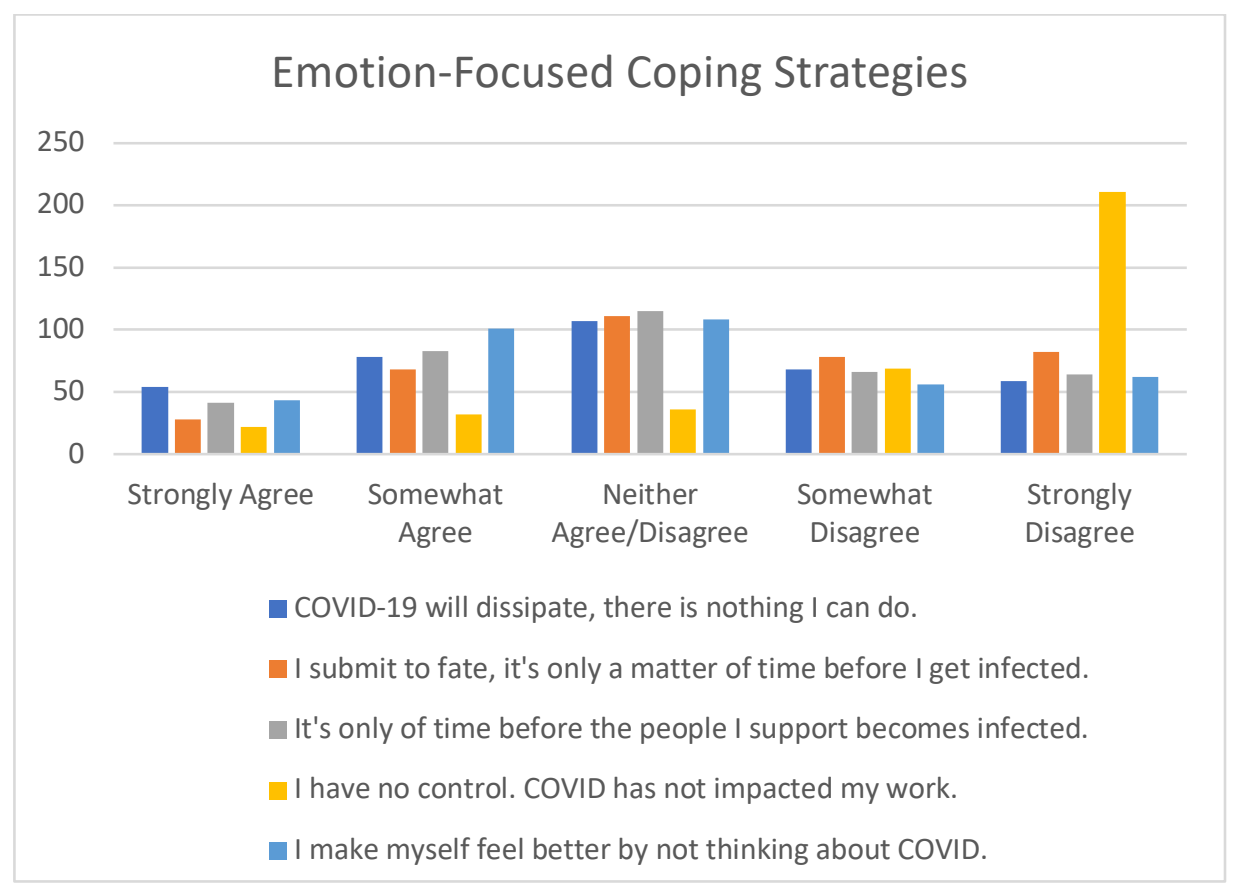

Figure 5: Emotion-focused strategies of DSPs coping with COVID-19, 5-point Likert Scale (1 = Strongly Agree, 5 = Strongly Disagree), $\mathrm{N}=486$

\section{DSP Comments Content Analysis}

The researchers analyzed all participants' responses using content analysis with a grounded theory approach to identify DSPs' perception of risk and coping strategies. This method allows for written or oral material to be classified into identified categories of similar meanings (Moretti et al., 2011). These categories represent either explicit or inferred communication (Hsieh \& Shannon, 2005). Abrahamson (1983) also emphasized that content analysis can be significantly used to examine essentially any kind of communication materials, including narrative responses, survey questions, interviews, focus groups, and observations because researchers can engage in data collection with or without direct contact with persons studied. Data were then inductively coded and subjected to Clarke and Braun's (2015) thematic analysis procedure.

\section{Table 4. DSP Comments Content Analysis}

\begin{tabular}{|l|c|c|}
\hline Category & Responses & Percentage (\%) \\
\hline Stated “no comment" & 4 & 5 \\
\hline COVID has not affected me & 7 & 10 \\
\hline PPE concerns & 5 & 7 \\
\hline Financial concerns & 4 & 5 \\
\hline Dealing with change & 3 & 4 \\
\hline Does not like how job handled COVID & 13 & 18 \\
\hline Worried/thought about death & 7 & 10 \\
\hline Fear of exposure/illness & 20 & 28 \\
\hline Liked how job handled COVID & 3 & 4 \\
\hline Miscellaneous & 6 & $\mathbf{8 \%}$ \\
\hline Total & $\mathbf{7 2}$ & $\mathbf{9 9 \%}$ \\
\hline
\end{tabular}




\section{Discussion}

The purpose of our study was to explore the DSPs' perception of the risk of COVID-19 infection while providing services to people with IDD. We also examined emotional and problem-solving coping strategies that DSPs may have used to deal with the pandemic. The study was driven by two research questions: (1) how do DSPs perceive the risk of being infected by COVID-19 while providing care and services to people with IDD? and (2) how do DSPs cope with the risk of being infected with COVID-19 and with the pandemic? Once the data analysis was complete, the data were organized to provide answers to the two research questions. Regarding the first question (the risk of being infected), data revealed that DSPs perceived they were at risk for contracting COVID-19 while providing services to people with IDD. In addition, DSPs also believed that the people they supported were more at risk of contracting COVID-19 because they have IDD. Data also showed that DSPs understood that COVID-19 was a global crisis and believed that the infection would not dissipate and would continue to compromise their health and safety as well as the health and safety of those they supported. DSPs perceived that COVID-19 was not going to go away, and they experienced fear of infection. DSPs worried continuously that they would become infected with COVID-19, although they were given proper PPE and were trained to reduce the risk of infection.

The second research question (coping with the risk of infection) described the coping strategies to determine whether the DSPs engaged in problem-focused strategies or emotion-focused strategies to cope with COVID19. The data revealed that DSPs engaged in more problem-focused strategies than emotion-focused strategies to cope with their perception of COVID-19. These problem-focused strategies included DSPs listening to their supervisors and engaging in practices to reduce risk of infection. Those that engaged in emotion-focused coping strategies believed they had no control over the virus and would often try not to think about it.

\section{Transactional Model of Stress and Coping}

Lazarus and Folkman (1984) theorized that stressful events will trigger an innate appraisal process in which the individual assesses the degree of threat in relation to their well-being. When an event is perceived as threatening, such as the current COVID-19 crisis, a secondary appraisal process provides a global assessment of the individual's coping resources and ability to manage that threat. The ability to effectively manage that stressful event depends on one's effectiveness to cognitively engage in coping strategies. Folkman and Lazarus (1991) described coping as a complex, organized sequence of behaviors that include cognitive appraisal and impulsive reaction. Karasek (1979) further added that the experience of stress is a consequence of the interaction between the stressor (e.g., COVID-19 vs. job demands) and the individual's perception of control over the stressor (e.g., infection and job control). Thus, in this study, we note that DSPs already work a highly demanding job but, when that job is placed within a global pandemic, DSPs will experience additional stress, resulting in an automatic response of a coping mechanism. It is worth noting that we did not delve into the exact type of stressors. The underlying assumption was that COVID-19 was the primary stressor and the aim of this research was exploratory, to determine how DSPs coped with COVID-19.

\section{DSP Coping Strategies}

Lazarus and Folkman (1984) described problem-focused coping strategies as actions such as problem solving, gathering information, weighing options, and acting upon choice. These coping strategies are most often used when the situation is perceived as being manageable to change and social support is available (Holahan \& Moos, 1987). We found that more DSPs engaged in problem-solving strategies because they felt they had obtained and gathered enough information to engage in practices that would reduce the risk of contracting the COVID-19 infection.

In contrast, Lazarus and Folkman (1984) described emotion-focused coping strategies as actions that would lessen the emotional distress caused by external stressful events or situations that, for our study, was the 
COVID-19 pandemic. These coping strategies included avoidance, minimization, distancing, selective attention, positive comparisons, and extracting positive value from negative events. Our results demonstrated that some DSPs did engage in emotion-focused strategies because they believed they had no real control against risk of infection.

\section{Limitations of the Study}

Limitations were noted in this study. First, the study was conducted through an online Likert-scale survey with no modification to prior responses or in-depth probing. DSPs' responses, therefore, are less rich than what could have resulted from face-to-face interviews. In addition, the 30-day timeframe in which we collected data posed another limitation, restricting additional DSPs from participating in the study.

Cronbach's alpha coefficient was used to evaluate the internal consistency of the questionnaire. An alpha value equal to or higher than 0.70 is an acceptable value indicating good internal reliability. In our study, one of the five questionnaire sections showed poor internal reliability. For emotion-focused coping strategies, Cronbach's alpha measured at .57, indicating poor internal reliability. Removing one item did not improve its reliability, showing that the items collectively were not a good measure of emotion-focused coping. In addition, the questionnaire was administered twice to ensure reliability of responses over time; however, due to a programming error, scores were unable to be extracted for individual participants for both occasions. Therefore, test-retest reliability could not be assessed using the data available.

Despite these limitations, our study adds to the literature that demonstrates how a crisis and the perception of that crisis play a role in how DSPs cope to maintain their work expectations. Through this COVID-19 pandemic, DSPs cope not only per their ability to effectively manage the stresses that already come with their job but also on their effectiveness to cognitively process the COVID-19 pandemic.

\section{Recommendations}

There is still much to learn about COVID-19 and, as a second wave of infection rates begins, the government will continue to innovate ways to control and contain the virus, in hopes a vaccine becomes available. It is important to continue protecting DSPs by having current, accurate, and accessible health information on the virus to ensure they have necessary information for protection. Such information can be provided through various platforms, such as organizational COVID-19 hotlines. By establishing COVID-19 informational phone services, organizations can provide a way for DSPs and other organizational employees to call in with questions, providing information but also perhaps some peace of mind. In addition, having ready and accessible materials in service programs and residential group homes could benefit DSPs and the people they support. DSPs can educate themselves and review with the residents in the group home or service program. This material should also include accessible means of communication to people with disabilities, such as Braille or PECS (Picture Exchange Communication Systems) that may make it easier for people with IDD to understand. COVID-19 guidelines and safety procedures should always be reviewed to minimize risk of infection not only among DSPs but also among the people they support.

Our study also points to the emerging need to address DSP social and emotional well-being. IDD service provider organizations can enhance resilience by offering health and wellness platforms to the DSPs, such as offering routine virus and symptom-testing opportunities, medical telehealth services, virtual social chat rooms, virtual exercise classes, and/or movie or game nights that would engage DSPs and the people they support. Providing virtual medical and social supports reduces the impact of isolation for both DSPs and people with IDD. Further, such services would improve DSPs' capacity to cope with COVID-19 by easing their anxiety, fears of infection, and isolation. 
Our study also demonstrated that DSPs feared a risk that the people they supported would become infected with COVID-19 and that the DSPs would have to assist in obtaining medical care. While the shortage of critical care and medical equipment is lessening, Wright (2020) described several adults who are deaf and blind reporting concerns of inherent biases they faced in receiving equitable medical treatment. In addition, several states adopted crisis standards-of-care guidelines that excluded people with IDD from accessing medical resources (Ne'eman, 2020). Thus, IDD provider organizations would benefit from establishing guidelines and connections with medical networks to ensure a process for care and treatment. By establishing connections and relationships with local medical facilities, collaborative processes can be established to treat people with IDD while ensuring equitable medical services. Such collaborative networks and processes can be considered as "reasonable accommodations" under the Americans with Disabilities Act, thus increasing protections for people with IDD and their DSPs.

\section{Implications for Practice}

Our study demonstrated the need for IDD service provider organizations to continue updating accessible health information and organizational practices that would protect DSPs from contracting COVID-19. IDD provider organizations need to institute COVID-19 informational services so DSPs can have a resource when they want COVID-related information. Having readily accessible information in platforms such as organizational COVID-19 hotlines, information posted on their websites, or by simply having COVID-related monthly meetings can impact DSPs' understanding to effectively cope with the stress of the pandemic while on the job. Thus, IDD service providers should consider assigning specific administrative employees or hiring new employees for the development of a COVID-19 task force. These employees will be tasked to ensure COVID-19 health-related information is accessible to all organizational employees including DSPs. This task force can also work to gather new and updated information on COVID-19 to disseminate to the organization. In addition, they can work on evaluating organizational procedures to ensure organizational practices are consistent with COVID-19 health-related guidelines, upkeep information and resources on organizational websites, and address employee COVID-19-related questions and concerns. With a group of employees designated to focus on COVID-19, resources and information will be streamlined throughout the organization, eliminating confusion and misinformation.

Our study also demonstrated the need for IDD provider organizations to address DSPs' own health and safety. IDD service provider organizations can enhance DSPs' emotional and mental health by offering health initiatives that include programs for routine COVID-19 testing and medical care, including available COVID19 testing locations, virtual medical services, or medical networks that would assess and treat DSPs. These health initiatives can be developed through managed care contracts. In Medicaid managed care contracts, the IDD provider organizations work with managed care organizations (MCOs) in creating agreements with healthcare professionals or healthcare facilities to deliver specified services. The aim of MCOs is to reduce program costs and provide better utilization of health services through the contracting of services. Thus, our study data can be used by IDD service providers to negotiate MCO contracts with appropriate costs to services that include COVID-19 testing locations, virtual medical services, and mental health services. In addition, IDD provider organizations can negotiate contracted services with local medical facilities for services that can include measuring the quality of care or timeliness of care response to people with disabilities and their DSPs. They can identify those at a higher risk due to chronic medical conditions for care and response prioritization. A list of social services available can be offered to DSPs experiencing COVID-related hardships. Such services may not only result in more expedient testing and diagnosis that would ultimately reduce further risk of exposure and spreading of infection, but it may also impact how DSPs cope with COVID-19 (Rosenbaum \& Velasquez, 2020). Knowing that there are accessible COVID-19-related services will provide the DSPs with a sense of ease and peace of mind knowing their IDD provider organization will be there to provide the 
necessary information to obtain services needed. Through these organizational health initiatives, DSPs will not only feel the support from their organization but will also feel protected and safe.

\section{Conclusion}

DSPs and people with IDD represent a diverse group with varying needs in responding to COVID-19. As we move further along in the pandemic's trajectory, we need to prioritize new supports, resources, and treatments. People with IDD rely on assistance with personal care, may have difficulty communicating symptoms of illness, resulting in delays in diagnosis and, thus, increasing the spread and risk of infection. Therefore, people with IDD and their essential workers, DSPs, are at higher risk of contracting the virus due to the inability to maintain physical distancing. Organizations must be present and have information and support to mitigate potential risk and reduce stress. We are now entering another phase of the pandemic. DSPs may still need to shelter-in-place with the people they support for long periods at a time. Organizations must be prepared to plan services and social supports to meet the safety, mental health, and well-being of all DSPs. Because of the changing and rapid nature of COVID-19, it is critical that service provider organizations periodically reassess procedures to address the needs of DSPs to incorporate practices and services that will ease DSPs' stress, concerns, and questions, which will ultimately lead to improved support of people with IDD. 


\section{References}

Abrahamson, M. (1983). Social research methods. Prentice Hall.

Chang, T.-Z., \& Vowles, N. (2013). Strategies for improving data reliability for online surveys: A case study. International Journal of Electronic Commerce Studies, 4(1), 121-130. http://academicpub.org/ojs/index.php/ijecs/article/view/1121

Clarke, V., \& Braun, V. (2016). Thematic analysis: Providing accessible guidance on doing and understanding. The Journal of Positive Psychology, 12(3), 297-298. https://doi.org/10.1080/17439760.2016.1262613

Denscombe, M. (1998). The good research guide: For small-scale social research projects. Open University Press.

Dixon-Ibarra, A., \& Horner-Johnson, W. (2014). Disability status as an antecedent to chronic conditions: National health interview survey, 2006-2012. Preventing Chronic Disease: Public Health Research, Practice, and Policy, 11, E15. https://doi.org/10.5888/pcd11.130251

Folkman, S., \& Lazarus, R. S. (1991). Coping and emotion. In A. Monat \& R. S. Lazarus (Eds.), Stress and coping: An anthology (pp. 207-227). Columbia University Press. (Reprinted from "Psychological and Biological Approaches to Emotion," edited by N. Stein, B. Leventhal, and T. Trabasso, 1990, Lawrence Erlbaum Associates, Inc)

Holahan, C. J., \& Moos, R. H. (1987). Personal and contextual determinants of coping strategies. Journal of Personality and Social Psychology, 52(5), 946-955. https://psycnet.apa.org/buy/1987-25245-001

Hsieh, H. F., \& Shannon, S. E. (2005). Three approaches to qualitative content analysis. Qualitative Health Research, 15(9), 1277-1288. https://doi.org/10.1177/1049732305276687

Karasek, R. A., Jr. (1979). Job demands, job decision latitude, and mental strain: Implications for job redesign. Administrative Science Quarterly, 24(2), 285-308. https://doi.org/10.2307/2392498

Kimball, A., Hatfield, K. M., Aarons, M., James, A., Taylor, J., Spicer, K., Bardossy, A. C., Oakley, L. P., Tanwar, S., Chisty, Z., Bell, J. M., Methner, M., Harney, J., Jacobs, J. R., Carlson, C. M., McLaughlin, H. P., Stone, N., Clark, S., Brostrom-Smith, C., ...Jernigan, J. A. (2020). Asymptomatic and presymptomatic SARS-CoV-2 infections in residents of a long-term care skilled nursing facility-King County, Washington, March 2020. Morbidity and Mortality Weekly Report, 69(13), 377-381. https://www.ncbi.nlm.nih.gov/pmc/articles/PMC7119514/

Lazarus, R. S., \& Folkman, S. (1984). Stress, appraisal, and coping. Springer.

Moretti, F., van Vliet, L., Bensing, J., Deledda, G., Mazzi, M., Rimondini, M., Zimmerman, C., \& Fletcher, I. (2011). A standardized approach to qualitative content analysis of focus group discussions from different countries. Patient Education and Counseling, 82(3), 420-428. https://doi.org/10.1016/j.pec.2011.01.005

Ne'eman, A. (2020). When it comes to rationing, disability rights law prohibits more than prejudice. In Hastings Bioethics Forum. https://www.thehastingscenter.org/when-it-comes-to-rationingdisability-rights-law-prohibits-more-than-prejudice/

Okoro, C. A., Hollis, N. D., Cyrus, A. C., \& Griffin-Blake, S. (2018). Prevalence of disabilities and health care access by disability status and type among adults-United States, 2016. Morbidity and Mortality Weekly Report, 67(32), 882-887. https://www.cdc.gov/mmwr/volumes/67/wr/mm6732a3.htm?s_cid = mm6732a3_w 
Park, C. L., Aldwin, C. M., Fenster, J. R., \& Snyder, L. B. (2008). Pathways to posttraumatic growth versus posttraumatic stress: Coping and emotional reactions following the September 11, 2001, terrorist attacks. American Journal of Orthopsychiatry, 78(3), 300-312. https://doi.org/10.1037/a0014054

Rosenbaum, S., \& Velasquez, M. (2020). States are using Medicaid managed care to address the COVID-19 pandemic. The Commonwealth Fund. https://doi.org/10.26099/1wbv-5b6o

Stevens, A., Courtney-Long, E., Gillespie, C., \& Armour, B. S. (2014). Hypertension Among US Adults by Disability Status and Type, National Health and Nutrition Examination Survey, 2001-2010. Preventing Chronic Disease, 11, E139. https://www.ncbi.nlm.nih.gov/pmc/articles/PMC4133509/

Wright, K. B. (2005). Researching Internet-based populations: Advantages and disadvantages of online survey research, online questionnaire authoring software packages, and web survey services. Journal of Computer-Mediated Communication, 10 (3). https://doi.org/10.1111/j.1083-6101.2005.tboo259.x

Wright, R. (2020). Who is “worthy"? Deaf-blind people fear that doctors won't save them from the Coronavirus. The New Yorker. https://www.newyorker.com/news/our-columnists/who-is-worthydeaf-blind-people-fear-that-doctors-wont-save-them-from-the-coronavirus

Zakowski, S. G., Hall, M. H., Klein, L. C., \& Baum, A. (2001). Appraised control, coping, and stress in a community sample: A test of the goodness-of-fit hypothesis. Annals of Behavioral Medicine, 23(3), 158-165. https://doi.org/10.1207/S15324796ABM2303_3 issues. JSBHS articles include peer-reviewed research reports, brief reports, comprehensive literature reviews, books reviews, and student research. 